T. Higa

Nagoya Math. J.

Vol. 96 (1984), 41-60

\title{
ON THE TOPOLOGICAL STRUCTURE OF AFFINELY CONNECTED MANIFOLDS
}

\author{
TATSUO HIGA
}

\section{Introduction}

The purpose of the present paper is to investigate the relationship between the topological structure and differential geometric objects for affinely connected manifolds.

Let $M$ be a compact, connected and oriented Riemannian manifold, $P^{r}(M)$ the vector space of all parallel $r$-forms on $M$ and $b_{r}(M)$ the $r$-th Betti number of $M$. Since every parallel form is harmonic, it follows from the Hodge-de Rham theory that the inequality $\operatorname{dim} P^{r}(M) \leqq b_{r}(M)$ holds for all $r=1, \cdots, \operatorname{dim} M$ (cf. [3], [5]). We shall generalize these inequalities to compact affinely connected manifolds.

Next, let $M$ be a non-compact manifold. A connected submanifold $N$ of $M$ is called a soul if $\operatorname{dim} N<\operatorname{dim} M$ and if the inclusion $i: N \rightarrow M$ is a homotopy equivalence. J. Cheeger and D. Gromoll proved the following remarkable theorem. If $M$ is a complete Riemannian manifold with nonnegative sectional curvature then $M$ has a compact soul (see [1] Theorem 1.11 and 2.1). We shall give another kind of sufficient conditions for $M$ to have a (compact) soul.

Finally, a connected manifold $M$ is said to be reducible if $M$ is diffeomorphic to a product manifold $M_{1} \times M_{2}$ with $\operatorname{dim} M_{i} \geqq 1, i=1,2$. Otherwise, $M$ is said to be irreducible. We shall find a differential geometric condition for $M$ to be reducible. We note that de Rham's Decomposition Theorem ([2]) furnishes a prototype for this condition (for irreducible manifolds, see [8]).

In order to obtain our results in a unified manner, we introduce certain family of functions on a connected manifold $M$ with an affine connection $\Gamma$. A function $f$ on $M$ is called an affine function if, for every geodesic $c(t)$ with an affine parameter $t$, there are real constants $a$ and $b$

Received July 8, 1983. 
such that $f(c(t))=a t+b$ for all $t$. We can regard each affine function as a "harmonic mapping". In fact, if $\Gamma$ is symmetric, then every affine function satisfies formally the defining equation of harmonic mappings (see [4] p. 116).

It is shown that the set $A(M, \Gamma)$ of all affine functions on $M$ is a finite-dimensional real vector space and satisfies $1 \leqq \operatorname{dim} A(M, \Gamma) \leqq \operatorname{dim} M$ + 1. By making use of $A(M, \Gamma)$, we shall define another finite-dimensional real vector space $V(M, \Gamma)$. Let $P^{1}(M, \Gamma)$ be the vector space of all parallel 1-forms on $M$ and $k(M)$ the non-negative integer defined to be the largest $k$ such that $H_{k}\left(M, Z_{2}\right) \neq 0$, where $H_{k}\left(M, Z_{2}\right)$ denotes the singular homology group of $M$ with coefficient $Z_{2}$. For simplicity, we suppose that $\Gamma$ is complete and symmetric. Then we can state our main results as follows.

A. If $M$ is compact, then $\operatorname{dim} P^{1}(M, \Gamma) \leqq b_{1}(M)$.

B. If $\operatorname{dim} A(M, \Gamma)>1$, then $M$ has a soul. Moreover, we have $k(M)$ $\leqq \operatorname{dim} M-\operatorname{dim} A(M, \Gamma)+1$. The equality holds if and only if $M$ has a compact soul $N$ with $\operatorname{dim} N=\operatorname{dim} M-\operatorname{dim} A(M, \Gamma)+1$.

C. If $m=\operatorname{dim} V(M, \Gamma)>0$, then there exists a totally geodesic submanifold $M^{\prime}$ of $M$ such that $M$ is diffeomorphic to $\boldsymbol{R}^{m} \times M^{\prime}$.

We remark that $M$ is not always affinely isomorphic to the product affinely connected manifold $\boldsymbol{R}^{m} \times M^{\prime}$. However, in the Riemannian case, we can prove more (see [7]).

In Section 1 and Section 2, we shall study some basic properties of affine functions. In Section 3, we shall state our main theorems in a rigorous form. The proof of the theorems will be given in Section 4. The crucial point of the proof lies in a careful use of geodesics. In the last section, we shall consider affine symmetric spaces and prove the following result. Let $M=G / H$ be an affine symmetric space with the canonical connection $\Gamma$ on $G / H$ (see [10] Chap. III). If $G$ is solvable and $M$ is simply connected, then $\operatorname{dim} A(M, \Gamma)>1$.

Throughout this paper, all manifolds and differential geometric objects on them are assumed to be differentiable of class $C^{\infty}$. For brevity's sake, we shall often use the adjective "smooth" instead of "differentiable".

\section{§1. Affine functions}

Let $M$ be a connected smooth manifold with an affine connection $\Gamma$. For a smooth curve $c(t)$ in $M$, we denote by $\dot{c}(t)$ the tangent vector to 
the curve at $c(t)$ and by $D \dot{c}(t) / d t$ the covariant derivative of $\dot{c}(t)$. A smooth curve $c(t)$ in $M$ defined on an open interval $I$ is called a geodesic if $D \dot{c}(t) / d t=0$ on $I$. If $c$ is a geodesic (as a point set), any parameter $t$ with respect to which $c=c(t)$ is a geodesic is called an affine parameter of $c$. In this paper, all geodesics under consideration are assumed to be parametrized by affine parameter. The connection $\Gamma$ is said to be complete if every geodesic can be extended to a geodesic $c(t)$ defined for all $t \in R$, where $R$ denotes the field of real numbers.

Definition 1.1. A smooth function $f$ on $M$ is called an affine function on $M$ if, for every geodesic $c(t)$, there are real constants $a$ and $b$ such that $f(c(t))=a t+b$ for any $t$ whenever it is defined.

This definition does not depend on the choice of an affine parameter $t$ because any other affine parameter $t^{\prime}$ is given by an affine transformation $t^{\prime}=c t+d$, where $c \neq 0$ and $d$ are real constants.

Proposition 1.1. Let $f$ be an affine function on $M$. If the differential $(d f)_{x}$ of $f$ at some point $x \in M$ vanishes, then $f$ is a constant function on $M$.

Proof. Let $N$ denote the subset of $M$ consisting of all points $y$ such that $(d f)_{y}=0$. Clearly, $N$ is non-empty and closed in $M$. Let us take any $y \in N$ and any geodesic $c(t)$ with $c(0)=y$. Then we can put $f(c(t))$ $=a t+b(a, b \in R)$. Hence we have

$$
a=\left.\frac{d}{d t} f(c(t))\right|_{t=0}=(d f)_{y}(\dot{c}(0))=0 .
$$

This means that $f$ is constant on every geodesic starting from the point $y$. Let $U$ be a convex neighborhood of $y$ (see [9] vol. 1, p. 149). Since every point of $U$ can be joined to $y$ by a geodesic segment, $f$ is constant on $U$. Thus $U \subset N$ and hence $N$ is open in $M$. Since $M$ is connected, we can conclude that $f$ is constant on $M$.

Let $A(M, \Gamma)$ denote the set of all affine functions of $M$. Then it is clear that $A(M, \Gamma)$ is a linear subspace of the real vector space of all smooth functions on $M$. Every real number can be identified with a constant function on $M$, so we get the natural inclusion $i: R \rightarrow A(M, \Gamma)$.

Proposition 1.2. $A(M, \Gamma)$ is finite-dimensional and satisfies $1 \leqq$ $\operatorname{dim} A(M, \Gamma) \leqq \operatorname{dim} M+1$. Moreover, if $M$ is compact, then $\operatorname{dim} A(M, \Gamma)$ $=1$. 
Proof. Let us fix a point $x$ of $M$. Let $F: A(M, \Gamma) \rightarrow T_{x}^{*}(M)$ denote the linear mapping given by $F(f)=(d f)_{x}(f \in A(M, \Gamma))$, where $T_{x}^{*}(M)$ is the cotangent space to $M$ at $x$. Then Proposition 1.1 implies that the sequence $0 \rightarrow R \stackrel{i}{\rightarrow} A(M, \Gamma) \stackrel{F}{\rightarrow} T_{x}^{*}(M)$ is exact. This proves the first and second assertions. The last assertion follows easily from Proposition 1.1 and the fact that every smooth function on a compact manifold has a critical point.

Proposition 1.3. If $\Gamma$ is complete, then every bounded affine function $f$ on $M$ is a constant function on $M$.

Proof. Let $x \in M$ and let $c(t)(t \in R)$ be any geodesic with $c(0)=x$. Then we can put $f(c(t))=a t+b(a, b \in R)$. Now the function $t \mapsto|a t|$ on $\boldsymbol{R}$ is bounded, so $a=0$. Thus we have $(d f)_{x}(\dot{c}(0))=0$ and hence $(d f)_{x}=0$. Therefore the assertion follows immediately from Proposition 1.1.

Proposition 1.4. Let $1, f_{1}, \cdots, f_{n}$ be elements of $A(M, \Gamma)$. Then the following two statements are equivalent:

1) $1, f_{1}, \cdots, f_{n}$ are linearly independent in $A(M, \Gamma)$;

2) $d f_{1}, \cdots, d f_{n}$ are linearly independent at each point of $M$.

Proof. Suppose 1). Let $x$ be any point of $M$ and assume that $\sum_{i=1}^{n} a_{i}\left(d f_{i}\right)_{x}=0$ for real constants $a_{1}, \cdots, a_{n}$. Then we have $\left(d\left(\sum_{i=1}^{n} a_{i} f_{i}\right)\right)_{x}$ $=0$, so by Proposition 1.1 there is a constant $b$ such that $\sum_{i=1}^{n} a_{i} f_{i}+b$ $=0$. Hence we get $a_{i}=0$ for all $i$, which implies 2). The converse is obvious.

Now we set $a(M, \Gamma)=\operatorname{dim} A(M, \Gamma)-1$.

Proposition 1.5. Let $\boldsymbol{R}^{n}$ be the n-dimensional affine space with the standard flat affine connection $\Gamma_{0}$. Then we have $a\left(\boldsymbol{R}^{n}, \Gamma_{0}\right)=n$.

Proof. Let $\left(x_{1}, \cdots, x_{n}\right)$ be the canonical coordinate system on $\boldsymbol{R}^{n}$. Then the coordinate functions $x_{1}, \cdots, x_{n}$ belong to $A\left(\boldsymbol{R}^{n}, \Gamma_{0}\right)$. Moreover, it follows from Propositions 1.2 and 1.4 that $1, x_{1}, \cdots, x_{n}$ form a basis of $A\left(\boldsymbol{R}^{n}, \Gamma_{0}\right)$. Hence we have $a\left(\boldsymbol{R}^{n}, \Gamma_{0}\right)=n$.

Let $M^{\prime}$ be another connected smooth manifold with an affine connection $\Gamma^{\prime}$.

Definition 1.2 (cf. [11]). A smooth mapping $h: M \rightarrow M^{\prime}$ is said to be totally geodesic if, for every geodesic $c(t)$ of $M, h(c(t))$ is a geodesic of $M^{\prime}$. 
For a smooth mapping $h: M \rightarrow M^{\prime}$ and a smooth function $f$ on $M^{\prime}$, we denote by $h^{*}(f)$ the smooth function on $M$ given by $h^{*}(f)=f \circ h$. Then we have immediately the following proposition.

Proposition 1.6. If $h: M \rightarrow M^{\prime}$ is a totally geodesic mapping, then $h^{*}\left(A\left(M^{\prime}, \Gamma^{\prime}\right)\right) \subset A(M, \Gamma)$ and $h^{*}: A\left(M^{\prime}, \Gamma^{\prime}\right) \rightarrow A(M, \Gamma)$ is a linear homomorphism. If moreover $h$ is surjective, then $h^{*}: A\left(M^{\prime}, \Gamma\right) \rightarrow A(M, \Gamma)$ is injective.

Proposition 1.7. Let $M_{i}$ be a connected smooth manifold with an affine connection $\Gamma_{i}(i=1,2)$. Let $\Gamma_{1} \times \Gamma_{2}$ denote the product affine connection on $M_{1} \times M_{2}$. Then we have

$$
a\left(M_{1} \times M_{2}, \Gamma_{1} \times \Gamma_{2}\right)=a\left(M_{1}, \Gamma_{1}\right)+a\left(M_{2}, \Gamma_{2}\right) .
$$

Proof. For simplicity, we write $A=A\left(M_{1} \times M_{2}, \Gamma_{1} \times \Gamma_{2}\right)$ and $A_{i}=$ $A\left(M_{i}, \Gamma_{i}\right), i=1,2$. Since the natural projection $p_{i}: M_{1} \times M_{2} \rightarrow M_{i}$ is totally geodesic, $p_{i}^{*}: A_{i} \rightarrow A$ is an injective homomorphism $(i=1,2)$. Let us fix a point $\left(x_{0}, y_{0}\right)$ of $M_{1} \times M_{2}\left(x_{0} \in M_{1}, y_{0} \in M_{2}\right)$. Let $h_{i}: M_{i} \rightarrow M_{1} \times M_{2}$, $i=1,2$, denote the smooth mappings given by $h_{1}(x)=\left(x, y_{0}\right)\left(x \in M_{1}\right)$ and $h_{2}(y)=\left(x_{0}, y\right)\left(y \in M_{2}\right)$, respectively. Clearly, we have $h_{i}^{*}(A) \subset A_{i}, i=1,2$. For any $f \in A$, we set

$$
f=f-p_{1}^{*}\left(h_{1}^{*}(f)\right)-p_{2}^{*}\left(h_{2}^{*}(f)\right)+f\left(x_{0}, y_{0}\right) .
$$

Then $\tilde{f}$ lies in $A$ and satisfies $\tilde{f}\left(x_{0}, y_{0}\right)=0$. It is not hard to verify that $d \tilde{f}$ vanishes at $\left(x_{0}, y_{0}\right)$. It follows from Proposition 1.1 that $\tilde{f}$ vanishes identically on $M_{1} \times M_{2}$. Hence,

$$
f=p_{1}^{*}\left(h_{1}^{*}(f)\right)+p_{2}^{*}\left(h_{2}^{*}(f)\right)-f\left(x_{0}, y_{0}\right) .
$$

This formula means that $A=p_{1}^{*}\left(A_{1}\right)+p_{2}^{*}\left(A_{2}\right)$. Since $p_{1}^{*}\left(A_{1}\right) \cap p_{2}^{*}\left(A_{2}\right)$ consists of all constant functions on $M_{1} \times M_{2}$, it follows that $\operatorname{dim} A=\operatorname{dim} A_{1}$ $+\operatorname{dim} A_{2}-1$. This proves Proposition 1.7.

\section{§ 2. Parallel 1-forms and affine functions}

Let $M$ be a connected smooth manifold with an affine connection $\Gamma$. Let $T$ be the torsion tensor, $R$ the curvature tensor and $V$ the covariant differentiation of $\Gamma . \quad \Gamma$ is said to be symmetric if $T$ vanishes identically on $M$. Let $f$ be any smooth function on $M$. We set

$$
H_{f}(X, Y)=\left(\nabla_{X} d f\right)(Y)+\frac{1}{2} d f(T(X, Y))
$$


for all vector fields $X$ and $Y$ on $M$. Then it is easy to see that $H_{f}$ is a symmetric covariant 2-tensor on $M$.

LEMMA 2.1. For any smooth function $f$ on $M$ and any smooth curve $c(t)$ in $M$, we have

$$
\frac{d^{2}}{d t^{2}} f(c(t))=H_{f}(\dot{c}(t), \dot{c}(t))+d f\left(\frac{D \dot{c}(t)}{d t}\right) .
$$

Proof. For simplicity, let us denote by $F(t)$ the second derivative of $f(c(t))$ and set $H_{f}=H_{f}(\dot{c}(t), \dot{c}(t))$. We can assume that the curve $c(t)$ lies in a coordinate chart $\left(U,\left(y_{1}, \cdots, y_{m}\right)\right)$ of $M(m=\operatorname{dim} M)$. Let $\Gamma_{i j}^{k}, i, j, k$ $=1, \cdots, m$, denote the components of $\Gamma$ with respect to the coordinate system and set $c^{i}(t)=y_{i} \circ c(t), i=1, \cdots, m$. Then we have

$$
F(t)=\sum_{i, j=1}^{m} \frac{\partial^{2} f}{\partial y_{i} \partial y_{j}} \cdot \frac{d c^{i}}{d t} \cdot \frac{d c^{j}}{d t}+\sum_{k=1}^{m} \frac{\partial f}{\partial y_{k}} \cdot \frac{d^{2} c^{k}}{d t^{2}}
$$

and

$$
H_{f}=\sum_{i, j=1}^{m}\left(\frac{\partial^{2} f}{\partial y_{i} \partial y_{j}}-\frac{1}{2} \sum_{k=1}^{m}\left(\Gamma_{i j}^{k}+\Gamma_{j i}^{k}\right) \frac{\partial f}{\partial y_{k}}\right) \cdot \frac{d c^{i}}{d t} \cdot \frac{d c^{j}}{d t} .
$$

Hence $F(t)-H_{f}$ is given by

$$
\sum_{k=1}^{m} \frac{\partial f}{\partial y_{k}}\left(\frac{d^{2} c^{k}}{d t^{2}}+\sum_{i, j=1}^{m} \Gamma_{i j}^{k} \frac{d c^{i}}{d t} \cdot \frac{d c^{j}}{d t}\right),
$$

which proves the formula.

LEMMA 2.2. Let $f$ be a smooth function on $M$. If $d f$ is parallel, then $d f(T(X, Y))=0$ and $d f(R(X, Y) Z)=0$ hold for all vector fields $X, Y$ and $Z$ on $M$.

Proof. This can be obtained from the following simple calculations:

1) $\quad d f(T(X, Y))=d f\left(\nabla_{X} Y\right)-d f\left(\nabla_{Y} X\right)-d f([X, Y])$

$$
=X(d f(Y))-Y(d f(X))-d f([X, Y])=0 ;
$$

2) $d f(R(X, Y) Z)=d f\left(\nabla_{X} \nabla_{Y} Z\right)-d f\left(\nabla_{Y} \nabla_{X} Z\right)-d f\left(\nabla_{[X, Y]} Z\right)$

$$
=X Y(d f(Z))-Y X(d f(Z))-[X, Y](d f(Z))=0 .
$$

Proposition 2.1. Let $f$ be a smooth function on $M$. Then:

(1) $f$ is an affine function on $M$ if and only if $H_{f}$ vanishes identically on $M$;

(2) If $d f$ is a parallel 1-form, then $f$ is an affine function; 
(3) If $\Gamma$ is symmetric, then, for every $f \in A(M, \Gamma)$, df is a parallel 1-form.

Proof. (1) Suppose that $f$ is an affine function. Let $x$ be any point of $M$ and let $c(t)$ be any geodesic with $c(0)=x$. From Lemma 2.1, we have

$$
H_{f}(\dot{c}(0), \dot{c}(0))=\left.\frac{d^{2}}{d t^{2}} f(c(t))\right|_{t=0}=0
$$

and hence $H_{f}(u, u)=0$ for any $u \in T_{x}(M)$, where $T_{x}(M)$ denotes the tangent space to $M$ at $x$. Since $H_{f}$ is symmetric, we finally have $H_{f}(u, v)$ $=0$ for all $u, v \in T_{x}(M)$. This implies that $H_{f}$ vanishes on $M$. In a similar way, we can prove the converse.

(2) Since $d f$ is parallel, it follows from Lemma 2.2 that $H_{f}$ vanishes on $M$. Hence $f$ is an affine function.

(3) Since $\Gamma$ is symmetric, we have $\left(\nabla_{X} d f\right)(Y)=H_{f}(X, Y)=0$ for all vector fields $X$ and $Y$ on $M$, so $d f$ is parallel.

Remark. Let $M$ be a connected Riemannian manifold and let $f$ be a smooth function on $M$. Then $H_{f}$ is the Hessian of $f$ and the LaplaceBeltrami operator $\Delta$ is given by $\Delta f=$ Trace of $H_{f} . f$ is said to be harmonic if $\Delta f=0$. By Proposition 2.1(1), we can assert that every affine function on $M$ is harmonic.

Now we prove an inequality which gives a relation between $A(M, \Gamma)$ and the curvature of $M$. For any $x \in M$, let $\mathfrak{P}_{x}$ denote the linear subspace of $T_{x}^{*}(M)$ consisting of all covectors $\omega$ such that $\omega(R(X, Y) Z)=0$ for all $X, Y, Z \in T_{x}(M)$.

Proposition 2.2. If $\Gamma$ is symmetric, then we have $a(M, \Gamma) \leqq \operatorname{dim} \mathfrak{P}_{x}$ for any $x \in M$.

Proof. Let $F_{x}: A(M, \Gamma) \rightarrow T_{x}^{*}(M)$ denote the linear mapping given by $F_{x}(f)=(d f)_{x}(f \in A(M, \Gamma))$. Then, by Proposition 2.1(3) and Lemma 2.2, the image $F_{x}(A(M, \Gamma))$ is contained in $\mathfrak{P}_{x}$. Hence the sequence

$$
0 \longrightarrow R \stackrel{i}{\longrightarrow} A(M, \Gamma) \stackrel{F_{x}}{\longrightarrow} \mathfrak{P}_{x}
$$

is exact. This proves Proposition 2.2.

Proposition 2.3. For a given affine connection $\Gamma$ on $M$, there exists an affine connection $\tilde{\Gamma}$ on $M$ such that $\tilde{\Gamma}$ is symmetric and $A(M, \tilde{\Gamma})=$ 
$A(M, \Gamma)$. Moreover, if $\Gamma$ is complete, then $\tilde{\Gamma}$ can be taken so that it is complete.

Proof. For all vector fields $X$ and $Y$ on $M$, we set $\tilde{\nabla}_{X} Y=\nabla_{X} Y-$ $\frac{1}{2} T(X, Y)$. Then it is easy to see that $\tilde{\nabla}$ defines a desired affine connection on $M$ (cf. [9] vol. 1, p. 146).

\section{§3. The main theorems}

Let $M$ be a connected smooth manifold with an affine connection $\Gamma$, $A(M, \Gamma)$ the vector space of all affine functions on $M$ and $P^{1}(M, \Gamma)$ the vector space of all parallel 1-form of $M$. As before, we set $a(M, \Gamma)=$ $\operatorname{dim} A(M, \Gamma)-1$. Let $\tilde{W}$ denote the set of all vector fields $X$ on $M$ such that, for every $f \in A(M, \Gamma), X f$ is a constant function on $M$ and that $\nabla_{X} X=0$. We set

$$
W(M, \Gamma)=\left\{X \in \tilde{W} ; \nabla_{Y} X=0 \text { for all } Y \in \tilde{W}\right\}
$$

and

$$
W_{0}(M, \Gamma)=\{X \in W(M, \Gamma) ; X f=0 \text { for all } f \in A(M, \Gamma)\} .
$$

Then it is easy to see that $W(M, \Gamma)$ is a linear subspace of the real vector space of all vector fields on $M$ and that $W_{0}(M, \Gamma)$ is a linear subspace of $W(M, \Gamma)$. Hence we can define a real vector space $V(M, \Gamma)$ by $V(M, \Gamma)=$ $W(M, \Gamma) / W_{0}(M, \Gamma)$.

Proposition 3.1. If $\Gamma$ is symmetric, then every parallel vector field $X$ on $M$ belongs to $W(M, \Gamma)$.

Proof. Let $f$ be any element of $A(M, \Gamma)$. By Proposition 2.1(3), $d f$ is parallel. Hence $X f$ is a constant function on $M$. Since $X$ is parallel, we have $\nabla_{Y} X=0$ for all vector fields $Y$ on $M$. Therefore, $X$ belongs to $W(M, \Gamma)$.

Proposition 3.2. $\quad V(M, \Gamma)$ is finite-dimensional and satisfies dim $V(M, \Gamma) \leqq a(M, \Gamma)$.

Proof. We can assume that $n=a(M, \Gamma)>0$. Let $1, f_{1}, \cdots, f_{n}$ be a basis of $A(M, \Gamma)$ and let $F$ denote the linear mapping of $W(M, \Gamma)$ into $R^{n}$ defined by $F(X)=\left(X f_{1}, \cdots, X f_{n}\right)(X \in W(M, \Gamma))$. Then it is easy to verify that the kernel of $F$ coincides with $W_{0}(M, \Gamma)$. Hence we get $\operatorname{dim} V(M, \Gamma)$ $\leqq n$. 
Proposition 3.3. Let $M$ be a connected Riemannian manifold with metric tensor $g$ and $\Gamma$ the Riemannian connection of $M$. Then we have $\operatorname{dim} V(M, \Gamma)=a(M, \Gamma)$.

Proof. For any smooth function $f$ on $M$, we denote by grad $f$ the gradient of $f$. Namely, grad $f$ is a unique vector field on $M$ such that $g(\operatorname{grad} f, X)=d f(X)$ for any vector field $X$ on $M$. Let $f \in A(M, \Gamma)$. For all vector fileds $X$ and $Y$ on $M$, we have

$$
\begin{aligned}
g\left(\nabla_{X} \operatorname{grad} f, Y\right) & =X g(\operatorname{grad} f, Y)-g\left(\operatorname{grad} f, \nabla_{X} Y\right) \\
& =X(d f(Y))-d f\left(\nabla_{X} Y\right) \\
& =H_{f}(X, Y)
\end{aligned}
$$

and hence grad $f$ is a parallel vector field of $M$. For any $X \in W(M, \Gamma)$, let $[X] \in V(M, \Gamma)$ denote the coset determined by $X$. Let $n=a(M, \Gamma)$ and let $1, f_{1}, \cdots, f_{n}$ be a basis of $A(M, \Gamma)$. To prove Proposition 3.3, it suffices to verify that $\left[\operatorname{grad} f_{1}\right], \cdots,\left[\operatorname{grad} f_{n}\right]$ are linearly independent in $V(M, \Gamma)$. Assume now that $\sum_{i=1}^{n} a_{i}\left[\operatorname{grad} f_{i}\right]=0$ for real constants $a_{1}, \cdots$, $a_{n}$. If we set $f=\sum_{i=1}^{n} a_{i} f_{i}$, then $\operatorname{grad} f$ belongs to $W_{0}(M, \Gamma)$. Let $x \in M$ and let $\|v\|$ denote the norm of $v \in T_{x}(M)$. Then we have

$$
\left\|(\operatorname{grad} f)_{x}\right\|^{2}=(d f(\operatorname{grad} f))(x)=0
$$

and hence $(\operatorname{grad} f)_{x}=0$. It follows from Proposition 1.1 that there is a real constant $b$ such that $\sum_{i=1}^{n} a_{i} f_{i}+b=0$. Thus we get $a_{i}=0$ for all $i=1, \cdots, n$. This completes the proof of Proposition 3.3.

Let $H^{1}(M)$ be the first de Rham cohomology group of $M$ and $H_{*}\left(M, Z_{2}\right)$ the singular homology group of $M$ with coefficient group $Z_{2}=Z / 2 Z, Z$ being the module of all rational integers. We define a non-negative integer $k(M)$ by the following two conditions:

1) $H_{i}\left(M, Z_{2}\right)=0 \quad$ for all $i>k(M)$;

2) $H_{k}\left(M, Z_{2}\right) \neq 0 \quad$ for $k=k(M)$.

We are now in a position to state our main theorems, which will be proved in the next section.

THEOREM 3.4. Let $M$ be a connected smooth manifold with a symmetric affine connection $\Gamma$. Then there exist natural linear homomorphisms $j: A(M, \Gamma) \rightarrow P^{1}(M, \Gamma)$ and $k: P^{1}(M, \Gamma) \rightarrow H^{1}(M)$ such that the sequence

$$
0 \longrightarrow \boldsymbol{R} \stackrel{i}{\longrightarrow} A(M, \Gamma) \stackrel{j}{\longrightarrow} P^{1}(M, \Gamma) \stackrel{k}{\longrightarrow} H^{1}(M)
$$


is exact. Hence,

$$
0 \leqq \operatorname{dim} P^{1}(M, \Gamma)-a(M, \Gamma) \leqq \operatorname{dim} H^{1}(M) .
$$

In particular, if $M$ is compact, then $\operatorname{dim} P^{1}(M, \Gamma) \leqq b_{1}(M)$. Here $b_{1}(M)$ denotes the first Betti number of $M$.

TheOREm 3.5. Let $M$ be a non-compact connected smooth manifold with a complete affine connection $\Gamma$. Assume that $n=a(M, \Gamma)>0$. Then there exists a totally geodesic surjective submersion $\pi: M \rightarrow R^{n}$ with the following properties:

(1) every fibre $N_{a}=\pi^{-1}(a)\left(a \in \boldsymbol{R}^{n}\right)$ is a connected totally geodesic submanifold of $M$;

(2) for every $a \in \boldsymbol{R}^{n}$, the inclusion $i_{a}: N_{a} \rightarrow M$ is a homotopy equivalence;

(3) if $N_{b}$ is compact for some $b \in R^{n}$, then so is $N_{a}$ for every $a \in \boldsymbol{R}^{n}$;

(4) if $M$ is non-orientable, then so is $N_{a}$ for every $a \in \boldsymbol{R}^{n}$. Moreover, if $\pi^{\prime}: M \rightarrow \boldsymbol{R}^{n}$ is another totally geodesic surjective submersion, then there exists an affine transformation $T$ of $\boldsymbol{R}^{n}$ such that $\pi^{\prime}=T \circ \pi$.

We remark that if $\Gamma$ is symmetric then $\pi$ is an affine mapping (see [11]). Let $(x, y)$ be the canonical coordinate system on $R^{2}$ and set $M=$ $\boldsymbol{R}^{2}-\{(-1,0),(1,0)\}$. Then we have $H_{1}(M, Z) \cong Z \oplus Z$. Let $p: M \rightarrow \boldsymbol{R}$ denote the smooth function given by

$$
p(x, y)=\log \left((x-1)^{2}+y^{2}\right)-\log \left((x+1)^{2}+y^{2}\right) .
$$

Then $p$ is a surjective submersion. The fibre $p^{-1}(0), 0$ being the origin of $\boldsymbol{R}$, is a line, while any other fibre $p^{-1}(a)(a \in R, a \neq 0)$ is a circle. Therefore, this shows that the existence of surjective submersion does not always imply (2) or (3) of Theorem 3.5. It should be also remarked that $M$ has no soul.

Theorem 3.6. Let $M$ and $\Gamma$ be as in Theorem 3.5. Then we have

$$
k(M) \leqq \operatorname{dim} M-a(M, \Gamma) .
$$

The equality holds if and only if there exists a compact connected totally geodesic submanifold $N$ of $M$ such that

1) $\operatorname{dim} N=\operatorname{dim} M-a(M, \Gamma)$;

2) the inclusion $i: N \rightarrow M$ is a homotopy equivalence.

Theorem 3.7. Let $M$ and $\Gamma$ be as in Theorem 3.5 and let $n=\operatorname{dim} M$. If $a(M, \Gamma)=n$, then $M$ is diffeomorphic to $\boldsymbol{R}^{n}$. Assume further that $\Gamma$ is 
symmetric. Then $M$ is affinely isomorphic to $\boldsymbol{R}^{n}$ if and only if $a(M, \Gamma)=n$.

Theorem 3.8. Let $M$ and $\Gamma$ be as in Theorem 3.5. Assume that $m$ $=\operatorname{dim} V(M, \Gamma)>0$. Then there exists a connected totally geodesic submanifold $M^{\prime}$ of $M$ such that $M$ is diffeomorphic to the product manifold $\boldsymbol{R}^{m} \times M^{\prime}$. Moreover, $M^{\prime}$ is compact if and only if $k(M)=\operatorname{dim} M-m$.

We remark that there is a connected manifold $M$ with a complete and symmetric affine connection $\Gamma$ satisfying the following inequalities:

$$
0<\operatorname{dim} V(M, \Gamma)<a(M, \Gamma)<\operatorname{dim} M .
$$

Let $M$ be a connected complete Riemannian manifold and $\Gamma$ the Riemannian connection of $M$. From Proposition 3.3, we have $\operatorname{dim} V(M, \Gamma)$ $=a(M, \Gamma)$. In this case, we can prove more: There exists a connected Riemannian manifold $M^{\prime}$ such that $M$ is isometric to the Riemannian product $\boldsymbol{R}^{n} \times M^{\prime}$ of the standard Euclidean space $\boldsymbol{R}^{n}$ and $M^{\prime}$, where we put $n=a(M, \Gamma)$. We shall prove this theorem in [7].

\section{§4. Proof of the main theorems}

We keep the notations in Section 3. First of all, we prove Theorem 3.4. Let $\Gamma$ be a symmetric affine connection on $M$. Then, by Proposition 2.1(3), we can define a linear mapping $j: A(M, \Gamma) \rightarrow P^{1}(M, \Gamma)$ by $j(f)=d f$ $(f \in A(M, \Gamma))$. Since every parallel 1 -form $\omega$ is closed, it determines a cohomology class $k(\omega) \in H^{1}(M)$. Thus we get the linear mapping $k: P^{1}(M, \Gamma)$ $\rightarrow H^{1}(M)$ and the sequence:

$$
0 \longrightarrow R \stackrel{i}{\longrightarrow} A(M, \Gamma) \stackrel{j}{\longrightarrow} P^{1}(M, \Gamma) \stackrel{k}{\longrightarrow} H^{1}(M) .
$$

To prove the exactness of the sequence, it suffices to verify the relation $\operatorname{Ker} k \subset \operatorname{Im} j$. Let $\omega$ be any element of Ker $k$. Then there is a smooth function $f$ on $M$ such that $\omega=d f$. By Proposition 2.1(2), $f$ lies in $A(M, \Gamma)$ and hence $\omega=j(f) \in \operatorname{Im} f$. If $M$ is compact, then every $f \in A(M, \Gamma)$ is constant on $M$ (Proposition 1.2). This means that the sequence

$$
0 \longrightarrow P^{1}(M, \Gamma) \stackrel{k}{\longrightarrow} H^{1}(M)
$$

is exact. Hence we have $\operatorname{dim} P^{1}(M, \Gamma) \leqq b_{1}(M)$. We have thereby proved Theorem 3.4.

To prove Theorem 3.5, we need some lemmas. 
Let $M$ be a non-compact connected manifold with a complete affine connection $\Gamma$. Assume that $n=a(M, \Gamma)>0$. Let us fix a basis $1, f_{1}, \cdots$, $f_{n}$ of $A(M, \Gamma)$ and define a smooth mapping $\pi: M \rightarrow R^{n}$ by $\pi(x)=\left(f_{1}(x)\right.$, $\left.\cdots, f_{n}(x)\right)(x \in M)$. Then it is clear that, for every geodesic $c(t)(t \in R)$, there are two elements $a$ and $b$ of $R^{n}$ such that $\pi(c(t))=a t+b$ for all $t \in \boldsymbol{R}$. This shows that $\pi$ is a totally geodesic mapping. Let us fix a Riemannian metric $g^{0}$ on $M$ and consider the vector fields $\operatorname{grad} f_{1}, \cdots, \operatorname{grad} f_{n}$ on $M$. Let $A_{i j}$ denote the function on $M$ given by $A_{i j}=g^{0}\left(\operatorname{grad} f_{i}\right.$, $\left.\operatorname{grad} f_{j}\right)(i, j=1, \cdots, n)$. From Proposition 1.4, it is easy to see that $\operatorname{grad} f_{1}, \cdots, \operatorname{grad} f_{n}$ are linearly independent at each point of $M$. Hence the $n \times n$ matrix $\left(A_{i j}(x)\right)$ is non-singular for every $x \in M$. Let $\left(B_{i j}\right)$ be the inverse matrix of $\left(A_{i j}\right)$. For any $a=\left(a_{1}, \cdots, a_{n}\right) \in \boldsymbol{R}^{n}$, we set

$$
X(a)=\sum_{i, j=1}^{n} a_{i} B_{i j} \operatorname{grad} f_{j} .
$$

Then $X(a)$ is a smooth vector field on $M$. As usual, we identify $\boldsymbol{R}^{n}$ with the tangent space $T_{a}\left(\boldsymbol{R}^{n}\right), a \in \boldsymbol{R}^{n}$, by the canonical absolute parallelism on $\boldsymbol{R}^{n}$.

Lemma 4.1. For any $a=\left(a_{1}, \cdots, a_{n}\right) \in R^{n}$ and any $x \in M$, we have $d f_{i}\left(X(a)_{x}\right)=a_{i}, i=1, \cdots, n$ and $\pi_{*}\left(X(a)_{x}\right)=a$.

Proof. Let $\left(x_{1}, \cdots, x_{n}\right)$ be the canonical coordinate system on $\boldsymbol{R}^{n}$. Then we have

$$
\begin{aligned}
\left(\pi_{*}\left(X(a)_{x}\right)\right)\left(x_{i}\right) & =d f_{i}\left(X(a)_{x}\right) \\
& =d f_{i}\left(\sum_{j, k=1}^{n} a_{j} B_{j k}(x)\left(\operatorname{grad} f_{k}\right)_{x}\right) \\
& =\sum_{j, k=1}^{n} a_{j} B_{j k}(x) A_{k i}(x) \\
& =a_{i}
\end{aligned}
$$

for all $i=1, \cdots, n$, which proves the formulas.

Let $T M$ be the tangent bundle of $M$ and exp: $T M \rightarrow M$ the exponential mapping of $M$. Let $G: R \times R^{n} \times M \rightarrow M$ denote the mapping given by $G(t, a, x)=\exp t X(a)_{x}\left(t \in R, a \in R^{n}, x \in M\right)$.

LEMMA 4.2. $G: R \times \boldsymbol{R}^{n} \times M \rightarrow M$ is smooth and satisfies

$$
\pi(G(t, a, x))=a t+\pi(x)
$$

for all $t \in R, a \in R^{n}$ and $x \in M$. 
Proof. We define a mapping $G_{0}: R \times \boldsymbol{R}^{n} \times M \rightarrow T M$ by $G_{0}(t, a, x)=$ $t X(a)_{x} \quad\left(t \in R, a \in \boldsymbol{R}^{n}, x \in M\right)$, so that $G=\exp \circ G_{0}$. Therefore, it suffices to prove that $G_{0}$ is smooth. But this can be easily checked by taking suitable local coordinate systems. Now we shall prove the second assertion. Since the curve $R \ni t \mapsto G(t, a, x) \in M$ is a geodesic, there is an element $b=b(a, x)$ of $R^{n}$ such that $\pi(G(t, a, x))=b t+\pi(x)$. Differentiating this with respect to $t$ at $t=0$, we have $b=\pi_{*}\left(X(a)_{x}\right)$ and hence $a=b$ by Lemma 4.1.

Lemma 4.3. $\pi: M \rightarrow \boldsymbol{R}^{n}$ is a surjective submersion.

Proof. It is clear from Proposition 1.4 that the rank of $\pi$ is equal to $n$ at each point of $M$. Let $x_{0}$ be a point of $M$ and let $a$ be any point of $\boldsymbol{R}^{n}$. Then, from Lemma 4.2, we have

$$
\pi\left(G\left(1, a-\pi\left(x_{0}\right), x_{0}\right)=a,\right.
$$

so $\pi$ is surjective.

Proof of Theorem 3.5. Consider the totally geodesic surjective submersion $\pi: M \rightarrow \boldsymbol{R}^{n}$ and set $N_{a}=\pi^{-1}(a)\left(a \in \boldsymbol{R}^{n}\right)$. For any $x \in N_{a}$ and any $v \in T_{x}\left(N_{a}\right)$, let $c(t)\left(t \in R^{n}\right)$ be the geodesic determined by $(x, v)$. Then we can put $\pi(c(t))=b t+a\left(b \in \boldsymbol{R}^{n}\right)$. Now we have

$$
b=\left.\frac{d}{d t} \pi(c(t))\right|_{t=0}=\pi_{*}(v)=0
$$

and hence $\pi(c(t))=a$ for all $t \in \boldsymbol{R}$. Thus $N_{a}$ is a totally geodesic submanifold of $M$.

By Lemma 4.2, $\pi\left(G(1, a-\pi(x), x)=a\right.$ holds for all $a \in \boldsymbol{R}^{n}$ and $x \in M$, so we can define a smooth mapping $r_{a}: M \rightarrow N_{a}$ by $r_{a}(x)=G(1, a-\pi(x), x)$ $(x \in M)$. We have easily $r_{a} \circ i_{a}(x)=x$ for any $x \in N_{a}$, where $i_{a}: N_{a} \rightarrow M$ is the inclusion. It follows that $N_{a}$ is connected. Let $H_{a}: R \times M \rightarrow M$ denote the smooth mapping given by $H_{a}(t, x)=G(t, a-\pi(x), x)(t \in R$, $x \in M)$. Then we have $H_{a}(0, x)=x$ and $H_{a}(1, x)=i_{a} \circ r_{a}(x)$ for all $x \in M$. Hence the mapping $i_{a} \circ r_{a}: M \rightarrow M$ is homotopic to the identity mapping of $M$. Therefore $i_{a}: N_{a} \rightarrow M$ is a homotopy equivalence. (More precisely, $N_{a}$ is a strong deformation retract of $M$.)

Suppose now that $N_{a}$ is orientable for some $a \in \boldsymbol{R}^{n}$. Let $\boldsymbol{e}_{1}, \cdots, \boldsymbol{e}_{n}$ be the canonical orthonormal basis of $\boldsymbol{R}^{n}$ and set $X_{i}=X\left(e_{i}\right), i=1, \cdots$, $n$. Then, from Lemma 4.1, it is easy to see that, for any $x \in N_{a}$ and any 
basis $v_{1}, \cdots, v_{p}$ of $T_{x}\left(N_{a}\right)\left(p=\operatorname{dim} N_{a}\right), v_{1}, \cdots, v_{p},\left(X_{1}\right)_{x}, \cdots,\left(X_{n}\right)_{x}$ form a basis of $T_{x}(M)$. Let $\omega$ be a non-vanishing continuous $p$-form on $N_{a}$ and let $m=\operatorname{dim} M$. For any $x \in N_{a}$, let $\Omega_{x}^{0}$ denote the $m$-covector of $T_{x}(M)$ defined by

$$
\Omega_{x}^{0}\left(v_{1} \wedge \cdots \wedge v_{p} \wedge\left(X_{1}\right)_{x} \wedge \cdots \wedge\left(X_{n}\right)_{x}\right)=\omega_{x}\left(v_{1} \wedge \cdots \wedge v_{p}\right)
$$

for all vectors $v_{1}, \cdots, v_{p}$ of $T_{x}\left(N_{a}\right)$. Let $E$ be the pull-back of $T M$ by the inclusion $i_{a}: N_{a} \rightarrow M$, i.e., $E=i_{a}^{\sharp} T M$. Then $\Omega_{x}^{0}\left(x \in N_{a}\right)$ defines a nonvanishing continuous cross section of $\Lambda^{m} E^{*}$, where $E^{*}$ is the dual bundle of $E$ and $\Lambda^{m} E^{*}$ the exterior product bundle of $E^{*}$. For any $y \in M$, we set $c_{y}(t)=H_{a}(t, y) \quad(t \in R)$, so that $c_{y}(0)=y$ and $c_{x}(1)=r_{a}(y) \in N_{a}$. Let $p\left(c_{y}\right)$ denote the parallel translation along the curve $c_{y}(t)(0 \leqq t \leqq 1)$. Thus $p\left(c_{y}\right)$ is a linear isomorphism of $T_{y}(M)$ onto $E_{x}\left(x=r_{a}(y)\right)$. Then $p\left(c_{y}\right)$ can be canonically extended to a smooth vector bundle homomorphism $p^{m}(c): \Lambda^{m} T M \rightarrow \Lambda^{m} E$. Now we define the $m$-covector $\Omega_{y}$ on $T_{y}(M)$ by $\Omega_{y}(V)=\Omega_{x}^{0}\left(p^{m}(c)(V)\right)\left(V \in \Lambda^{m} T_{y}(M), x=r_{a}(y)\right)$. Then it can be easily seen that $\Omega_{y}(y \in M)$ defines a non-vanishing continuous $m$-form on $M$. Hence $M$ is orientable. We have thereby proved (4) of Theorem 3.5.

Let $\pi^{\prime}: M \rightarrow \boldsymbol{R}^{n}$ be another totally geodesic surjective submersion. Let $\left(x_{1}, \cdots, x_{n}\right)$ be the canonical coordinate system on $\boldsymbol{R}^{n}$ and set $f_{i}^{\prime}=x_{i}$ 。 $\pi^{\prime}, i=1, \cdots, n$. Then $f_{1}^{\prime}, \cdots, f_{n}^{\prime}$ are affine functions on $M$ and linearly independent in $A(M, \Gamma)$. Hence there are a non-singular $n \times n$ matrix $\left(a_{i j}\right)$ and real numbers $b_{1}, \cdots, b_{n}$ such that $f_{i}^{\prime}=\sum_{j=1}^{n} a_{i j} f_{j}+b_{i}$ for all $i=1, \cdots, n$. This proves the last assertion of Theorem 3.5.

To prove (3) of Theorem 3.5, we need the following lemma.

Lemma 4.4. Let $N$ be a connected smooth manifold. Then we have $0 \leqq k(N) \leqq \operatorname{dim} N$. Moreover, $N$ is compact if and only if $k(N)=\operatorname{dim} N$.

Proof of Lemma 4.4. It is well-known that the singular homology group $H_{*}\left(N, Z_{2}\right)$ has the following properties:

1) $H_{q}\left(N, Z_{2}\right)=0$ for all $q>\operatorname{dim} N$;

2) If $N$ is non-compact, then $H_{p}\left(N, Z_{2}\right)=0$ for $p=\operatorname{dim} N$;

3) If $N$ is compact, then $H_{p}\left(N, Z_{2}\right) \cong Z_{2}$ for $p=\operatorname{dim} N$.

(For more details, see for example [6]). Now the lemma follows immediately from these properties.

We return to the proof of Theorem 3.5(3). Suppose that $N_{b}\left(b \in \boldsymbol{R}^{n}\right)$ is compact. Let $a$ be any point of $\boldsymbol{R}^{n}$. Then $N_{a}$ is homotopy equivalent 
to $N_{b}$. From Lemma 4.4, we have

$$
k\left(N_{a}\right)=k\left(N_{b}\right)=\operatorname{dim} N_{b}=\operatorname{dim} N_{a} .
$$

Hence $N_{a}$ is compact. This completes the proof of Theorem 3.5.

Proof of Theorem 3.6. By Theorem 3.5, there exists a connected totally geodesic submanifold $N$ of $M$ such that a) the inclusion $i: N \rightarrow M$ is a homotopy equivalence and b) $\operatorname{dim} N=\operatorname{dim} M-a(M, \Gamma)$. From a), b) and Lemma 4.4, we have

$$
k(M)=k(N) \leqq \operatorname{dim} N=\operatorname{dim} M-a(M, \Gamma) .
$$

Moreover, $N$ is compact if and only if $k(M)=\operatorname{dim} M-a(M, \Gamma)$. We have thereby proved Theorem 3.5.

Proof of Theorem 3.7. In view of Propositions 1.5 and 2.3, it will be sufficient to prove that if $\Gamma$ is symmetric and $a(M, \Gamma)=\operatorname{dim} M$ then $M$ is affinely isomorphic to $\boldsymbol{R}^{n}$. Accordingly, we assume that $\Gamma$ is symmetric and $a(M, \Gamma)=\operatorname{dim} M$. Let $n=\operatorname{dim} M$ and consider the surjective submersion $\pi: M \rightarrow \boldsymbol{R}^{n}$ (Lemma 4.3). In this case, $\pi$ is an immersion, so we can define a Riemannian metric $g$ on $M$ by $g=\pi^{*} d s^{2}$, where $d s^{2}$ denotes the standard Euclidean metric on $R^{n}$ and $\pi^{*}$ the codifferential of $\pi$. As before, let $\boldsymbol{e}_{1}, \cdots, \boldsymbol{e}_{n}$ be the canonical orthonormal basis of $\boldsymbol{R}^{n}$ and set $X_{i}=X\left(e_{i}\right), i=1, \cdots, n$. By Lemma $4.1, d f_{i}\left(X_{j}\right)$ is constant on $M(i, j=$ $1, \cdots, n)$. On the other hand, by Proposition $2.1(3), d f_{i}$ is a parallel 1-form of $M(i=1, \cdots, n)$. Hence, for any vector field $X$ on $M$, we have $d f_{i}\left(\nabla_{X} X_{j}\right)=X\left(d f_{i}\left(X_{j}\right)\right)=0$ for all $i, j=1, \cdots, n$. It follows that $X_{1}, \cdots, X_{n}$ are parallel vector fields. Hence,

$$
\left[X_{i}, X_{j}\right]=\nabla_{X_{i}} X_{j}-\nabla_{X_{j}} X_{i}=0 \quad(i, j=1, \cdots, n) .
$$

Let $\tilde{V}$ denote the covariant differentiation of the Riemannian connection of $(M, g)$. Since $g\left(X_{i}, X_{j}\right)$ is constant on $M$ for all $i, j=1, \cdots, n$ we have $g\left(\tilde{\nabla}_{X_{i}} X_{j}, X_{k}\right)=0, i, j, k=1, \cdots, n$, and hence $\tilde{\nabla}_{X_{i}} X_{j}=0, i, j=1, \cdots, n$ (cf. [9] vol. 1 p. 160). This means that $\Gamma$ coincides with the Riemannian connection of $(M, g)$. As $\Gamma$ is complete, $(M, g)$ is a complete Riemannian manifold. It therefore follows from a well-known theorem in [9] (vol. 1 p. 176, Theorem 4.6) that $\pi$ is an isometry of $M$ onto $R^{n}$. This completes the proof of Theorem 3.7.

Proof of Theorem 3.8. We begin with the following lemma. 
Lemma 4.5. Let $X$ be any element of $W(M, \Gamma)$. Then:

1) Every integral curve of $X$ is a geodesic of $M$;

2) $X$ is a complete vector field on $M$.

Proof. 1) follows immediately from the condition $\nabla_{X} X=0$.

2) Let $x(t)(|t|<\varepsilon, \varepsilon>0)$ be an integral curve of $X$. Since $\Gamma$ is complete, $x(t)$ can be extended to a geodesic $c(t)$ defined for all $t \in \boldsymbol{R}$. Let $I$ denote the subset of $\boldsymbol{R}$ consisting of all points $t$ such that $\dot{c}(t)=$ $X_{c(t)}$. Clearly, $I$ is non-empty and closed in $R$. Let $t_{0}$ be any point of $I$ and let $y(t)\left(\left|t-t_{0}\right|<\varepsilon^{\prime}, \varepsilon^{\prime}>0\right)$ be an integral curve of $X$ with $y\left(t_{0}\right)=c\left(t_{0}\right)$. Then $y(t)$ is a geodesic with the initial condition $\left(c\left(t_{0}\right), X_{c\left(t_{0}\right)}\right)$. Hence $c(t)$ must coincide with $y(t)$ on a small open neighborhood of $t_{0}$. This shows that $I$ is open in $R$ and hence $I=R$. Therefore every integral curve of $X$ can be extended to an integral curve defined for all $t \in R$. Hence $X$ is complete.

Let us set $m=\operatorname{dim} V(M, \Gamma)$ and $n=a(M, \Gamma)$. Then we have $m \leqq n$ (Proposition 3.2).

Lemma 4.6. We can choose $Y_{1}, \cdots, Y_{m} \in W(M, \Gamma)$ and $f_{1}, \cdots, f_{m} \in A(M, \Gamma)$ in such a way that

1) $1, f_{1}, \cdots, f_{m}$ are linearly independent in $A(M, \Gamma)$;

2) $d f_{i}\left(Y_{j}\right)=\delta_{i j}$ for all $i, j=1, \cdots, m$, where $\delta_{i j}$ denotes Kronecker's delta.

Proof. For any $Y \in W(M, \Gamma)$, let $[Y] \in V(M, \Gamma)$ denote the coset determined by $Y$. Then we can choose $Y_{1}, \cdots, Y_{m} \in W(M, \Gamma)$ so that $\left[Y_{1}\right]$, $\cdots,\left[Y_{m}\right]$ form a basis of $V(M, \Gamma)$. Let $1, g_{1}, \cdots, g_{n}$ be a basis of $A(M, \Gamma)$ and set $A_{i j}=d g_{i}\left(Y_{j}\right), i=1, \cdots, n, j=1, \cdots, m$. Then, by definition, $A_{i j}$ 's are constants. Assume that $\sum_{j=1}^{m} a_{j} A_{i j}=0$ for real constants $a_{1}$, $\cdots, a_{m}(i=1, \cdots, n)$. Then we have easily $\sum_{j=1}^{m} a_{j}\left[Y_{j}\right]=0$ and hence $a_{j}=0$ for all $j=1, \cdots, m$. This means that the rank of the $n \times m$ matrix $\left(A_{i j}\right)$ is equal to $m$. We can assume that the $m \times m$ matrix $\left(A_{i j}\right)_{1 \leq i, j \leq m}$ is non-singular. Let $\left(B_{i j}\right)_{1 \leq i, j \leq m}$ be the inverse matrix of $\left(A_{i j}\right)$ and set $f_{i}=\sum_{j=1}^{m} B_{i j} g_{j}, i=1, \cdots, m$. Then $1, f_{1}, \cdots, f_{m}$ are linearly independent. Moreover, we have

$$
d f_{i}\left(Y_{j}\right)=\sum_{k=1}^{m} B_{i k} d g_{k}\left(Y_{j}\right)=\sum_{k=1}^{m} B_{i j} A_{k j}=\delta_{i j}
$$

for all $i, j=1, \cdots, m$. This proves Lemma 4.6. 
From now on, we fix $Y_{1}, \cdots, Y_{m} \in W(M, \Gamma)$ and $f_{1}, \cdots, f_{m} \in A(M, \Gamma)$ with the properties listed in Lemma 4.6. Let $p: M \rightarrow \boldsymbol{R}^{m}$ denote the smoath mapping given by $p(x)=\left(f_{1}(x), \cdots, f_{m}(x)\right)(x \in M)$. For any $a=\left(a_{1}, \cdots, a_{m}\right)$ $\in \boldsymbol{R}^{m}$, let $Y^{a}$ denote the vector field given by $Y^{a}=\sum_{i=1}^{m} a_{i} Y_{i}$. By Lemma 4.5, $Y^{a}$ is a complete vector field on $M$. We denote by $F_{t}^{a}$ the 1-parameter family of diffeomorphisms generated by $Y^{a}$. Let $F: R \times R^{m} \times M \rightarrow M$ denote the mapping defined by $F(t, a, x)=F_{t}^{a}(x)\left(t \in R, a \in R^{m}, x \in M\right)$.

Lemma 4.7. $F: \boldsymbol{R} \times \boldsymbol{R}^{m} \times M \rightarrow M$ is smooth and satisfies

$$
p(F(t, a, x))=a t+p(x)
$$

for all $t \in \boldsymbol{R}, a \in \boldsymbol{R}^{m}$ and $x \in M$.

Proof. By Lemma 4.5, the curve $R \ni t \mapsto F(t, a, x) \in M$ is a geodesic, so we can write $F(t, a, x)=\exp t Y_{x}^{a}$. Therefore, we can prove Lemma 4.7 by the same reasoning as in Lemma 4.2.

Now we set $M^{\prime}=p^{-1}(0), 0$ being the origin of $R^{m}$. Then $M^{\prime}$ is a closed totally geodesic submanifold of $M$. Let $h$ denote the smooth mapping $\boldsymbol{R}^{m} \times M^{\prime} \rightarrow M$ given by $h(a, x)=F(1, a, x)=F_{1}^{a}(x)\left(a \in \boldsymbol{R}^{m}, x \in M^{\prime}\right)$. From Lemma 4.7, we have $p(F(-1, p(x), x))=0$ for any $x \in M$, so we can define the smooth mapping $q: M \rightarrow M^{\prime}$ by $q(x)=F(-1, p(x), x)(x \in M)$. For any $a \in \boldsymbol{R}^{m}$ and any $x \in M^{\prime}$, we have $p(h(a, x))=a$ and hence

$$
\begin{aligned}
(p \times q) \circ h(a, x) & =(p(h(a, x)), q(h(a, x))) \\
& =\left(a, F_{-1}^{a} \circ F_{1}^{a}(x)\right) \\
& =(a, x) .
\end{aligned}
$$

On the other hand, we have for any $y \in M$

$$
\begin{aligned}
h \circ(p \times q)(y) & =h(p(y), q(y)) \\
& =F_{1}^{a} \circ F_{-1}^{a}(y) \\
& =y,
\end{aligned}
$$

where we put $a=p(y)$. These results show that $h$ is a diffeomorphism of $R^{m} \times M^{\prime}$ onto $M$. The last assertion follows easily from Lemma 4.4. This completes the proof of Theorem 3.8.

\section{§5. Affine symmetric spaces}

A symmetric space is a triple $(G, H, s)$ consisting of a connected Lie group $G$, a closed subgroup $H$ of $G$ and an involutive automorphism $s$ of $G$ 
such that $H$ lies between the closed subgroup $G_{s}$ of all fixed points of $s$ and the identity component of $G_{s}$. Let us consider the coset space $G / H$ and let $s_{0}$ denote the diffeomorphism $g H \rightarrow s(g) H$ of $G / H$ onto itself. Then $G / H$ has a unique affine connection $\Gamma$ invariant under $s_{0}$ and under the natural left action of $G$ (see [10] $\S 15$ ). $\Gamma$ is called the canonical affine connection on $G / H$. Then $G / H$ turns out to be an affine symmetric space with respect to the canonical affine connection. Conversely, every affine symmetric space is expressed in this form. Let $\mathfrak{g}$ and $\mathfrak{h}$ be the Lie algebras of $G$ and $H$, respectively. Let $m$ denote the $(-1)$-eigenspace of the differential of $s$. Then we have the canonical decomposition

$$
\mathfrak{g}=\mathfrak{h}+\mathfrak{m} \quad \text { (direct sum). }
$$

TheOrem 5.1. Let $(G, H, s)$ be a symmetric space, $\mathfrak{g}=\mathfrak{h}+\mathfrak{m}$ the canonical decomposition of the Lie algebra of $G$ and $\Gamma$ the canonical affine connection on $M=G / H$. Then:

1) $a(M, \Gamma) \leqq \operatorname{dim} \mathfrak{m}-\operatorname{dim}[\mathfrak{m},[\mathfrak{m}, \mathfrak{m}]]$;

2) The equality holds if $M$ is simply connected.

Proof. We denote by $\mathrm{gl}(\mathrm{m})$ the Lie algebra of all linear endomorphisms of $\mathfrak{m}$ and by $\mathfrak{m}^{*}$ the dual vector space of $\mathfrak{m}$. Let $\rho: \mathfrak{h} \rightarrow \mathfrak{g l}(\mathfrak{m})$ denote the linear isotropy representation given by $\rho(X)(Y)=[X, Y](X \in \mathfrak{h}, Y \in \mathfrak{m})$. If we set $\mathfrak{h}^{\prime}=[\mathfrak{m}, \mathfrak{m}]$, then $\mathfrak{h}^{\prime}$ is an ideal of $\mathfrak{h}$. We remark here that the Lie subalgebra $\rho\left(\mathfrak{h}^{\prime}\right)$ of $\mathfrak{g l}(\mathfrak{m})$ can be identified with the Lie algebra of the linear holonomy group $L_{0}$ at the origin $0=H \in G / H$ (see [9] vol. 2 p. 232). Moreover, if $M=G / H$ is simply connected, $L_{0}$ is connected. Let $\rho^{*}: \mathfrak{h} \rightarrow$ $\mathfrak{g l}\left(\mathfrak{m}^{*}\right)$ denote the representation defined by

$$
\left(\rho^{*}(X) \omega\right)(Y)=-\omega(\rho(X)(Y)) \quad\left(X \in \mathfrak{h}, Y \in \mathfrak{m}, \omega \in \mathfrak{m}^{*}\right) .
$$

We set

$$
\tilde{\mathfrak{a}}=\left\{\omega \in \mathfrak{M}^{*} ; \rho^{*}(X) \omega=0 \text { for all } X \in \mathfrak{h}^{\prime}\right\} .
$$

Let $P^{1}(M, \Gamma)$ be the vector space of all parallel 1-form of $M$. From the above remarks, it can be easily seen that $\operatorname{dim} P^{1}(M, \Gamma) \leqq \operatorname{dim} \tilde{a}$ and that if $M$ is simply connected then $\operatorname{dim} P^{1}(M, \Gamma)=\operatorname{dim} \tilde{a}$. Let $a$ denote the linear subspace of $m$ consisting of all vectors $Y$ such that $\omega(Y)=0$ for all $\omega \in \tilde{a}$. Then we have $\operatorname{dim} \tilde{\mathfrak{a}}=\operatorname{dim} \mathfrak{m}-\operatorname{dim} \mathfrak{a}$. For simplicity, we write $\mathfrak{b}=\rho\left(\mathfrak{h}^{\prime}\right)(\mathfrak{m})$. Let $X \in \mathfrak{h}^{\prime}$ and $Y \in \mathfrak{m}$. For any $\omega \in \tilde{a}$, we have

$$
\omega(\rho(X)(Y))=-\left(\rho^{*}(X) \omega\right)(Y)=0,
$$


which implies that $\mathfrak{b}$ is a linear subspace of $\mathfrak{a}$. Hence,

$$
\operatorname{dim} \tilde{a} \leqq \operatorname{dim} \mathfrak{m}-\operatorname{dim} \mathfrak{b} .
$$

On the other hand, let $\tilde{\mathfrak{b}}$ denote the linear subspace of $\mathfrak{m}^{*}$ consisting of all $\omega \in \mathfrak{m}^{*}$ such that $\omega(Z)=0$ for any $Z \in \mathfrak{b}$. Then, as in the above case, we have $\tilde{\mathfrak{b}} \subset \tilde{a}$. Hence,

$$
\operatorname{dim} \tilde{a} \geqq \operatorname{dim} \mathfrak{m}-\operatorname{dim} \mathfrak{b} .
$$

We have thereby proved the formula: $\operatorname{dim} \tilde{\mathfrak{a}}=\operatorname{dim} \mathfrak{m}-\operatorname{dim} \mathfrak{b}$. Now Theorem 5.1 follows easily from Theorem 3.4.

Corollary 5.2. Let $(G, H, s)$ be a symmetric space and $\Gamma$ the canonical affine connection on $M=G / H$.

1) If $G$ is semisimple, then $a(M, \Gamma)=0$.

2) If $G$ is solvable and if $M$ is simply connected, then $a(M, \Gamma)>0$.

Proof. Let $\mathfrak{g}=\mathfrak{h}+\mathfrak{m}$ be the canonical decomposition. If we set $\mathfrak{g}^{\prime}=[\mathfrak{m}, \mathfrak{m}]+\mathfrak{m}$, then $\mathfrak{g}^{\prime}$ is an ideal of $\mathfrak{g}$. We have easily

$$
\left[\mathfrak{g}^{\prime}, \mathfrak{g}^{\prime}\right]=[\mathfrak{m}, \mathfrak{m}]+[\mathfrak{m},[\mathfrak{m}, \mathfrak{m}]] \quad \text { (direct sum) } \text {. }
$$

Suppose first that $\mathfrak{g}$ is semisimple. Then $\mathfrak{g}^{\prime}$ is also semisimple. Thus we get $\left[\mathfrak{g}^{\prime}, \mathfrak{g}^{\prime}\right]=\mathfrak{g}^{\prime}$ and hence $[\mathfrak{m},[\mathfrak{m}, \mathfrak{m}]]=\mathfrak{m}$. Suppose now that $\mathfrak{g}$ is solvable. Then $\mathfrak{g}^{\prime}$ is also solvable. Thus $\left[\mathfrak{g}^{\prime}, \mathfrak{g}^{\prime}\right] \subseteq \mathfrak{g}^{\prime}$ and hence $[\mathfrak{m},[\mathfrak{m}, \mathfrak{m}]] \subseteq \mathfrak{m}$. Therefore the assertions 1) and 2) follow from Theorem 5.1.

\section{REFERENCES}

[1] J. Cheeger and D. Gromoll, On the structure of complete manifolds of nonnegative curvature, Ann. of Math., 96 (1972), 413-443.

[2] G. de Rham, Sur la réductibilité d'un espace de Riemann, Comment. Math. Helv., 26 (1952), 328-344.

[ 3 ] G. de Rham, Variétés Différentiables, Hermann, Paris, 1955.

[ 4 ] J. Eells and J. H. Sampson, Harmonic mappings of Riemannian manifolds, Amer. J. Math., 86 (1964), 109-160.

[5] S. I. Goldberg, Curvature and Homology, Academic Press, New York, 1962.

[ 6 ] M. J. Greenberg, Lectures on Algebraic Topology, Benjamin, New York, 1967.

[ 7 ] T. Higa, On a splitting theorem for Riemannian manifolds, to appear in Comment. Math. Univ. St. Paul., (1984).

[8] T. Higa, On irreducible manifolds and geometric structures, (preprint).

[9] S. Kobayashi and K. Nomizu, Foundations of Differential Geometry, John Wiley, New York, vol. 1, 1963; vol. 2, 1969.

[10] K. Nomizu, Invariant affine connections on homogeneous spaces, Amer. J. Math., 76 (1954), 33-65.

[11] J. Vilms, Totally geodesic maps, J. Differential Geom., 4 (1970), 73-79. 
Department of Mathematics Faculty of Science Rikkyo University Tokyo 171

Japan 